\title{
A FRAMEWORK FOR RELIABILITY MODELING OF ELECTRONICS
}

\author{
Edward B. Hakim \\ (908) 544-2185 \\ Army Research Laboratory \\ Component Reliability Branch \\ Electronics \& Power Sources Directorate, AMSRL-EP-RA \\ Fort Monmouth, NJ 07703-5601, U.S.A. \\ Michael D. Osterman and Carl A. Rust \\ (301) 405-5323 \\ CALCE Electronic Packaging Research Center \\ University of Maryland \\ College Park, Maryland 20742, U.S.A.
}

\section{INTRODUCTION}

Reliability is often defined as the probability that a product will perform its intended function within specified tolerances under stated conditions for a given period of time. This concept of reliability as a probability, typically quantified by assessing the mean time between failures, implies that field failure is inevitable. This common paradigm of reliability has been exploited, as is evident from the poor failure-rate estimates for electronic components based on empirical models fit to field data where root cause of failure was not considered (as in, for example, MIL-HDBK-217). These failure models are typically not derived from or based on any physics or mechanics of failure, and as such, they do not give the designer any insight into or control over actual failure mechanisms. Thus, for designing and testing a new product or a product with new technologies, these models are inappropriate and misleading.

Using this empuncal approach, the mean time between failure (MTBF) for a system is derived from the reciprocal of the summation of component failure rates. When compared with actual test data, there is little or no correlation between the empirically predicted reliability and the actual observed reliability (see table 1).
Phyșics-of-failure is an approach to reliability modeling, design, and assessment that utilizes knowledge of failure mechanisms to prevent product failures through robust design and manufacturing practices. The approach is based on the identification of potential failure mechanisms and failure sites for the product. Each failure mechanism is described by the relations between the stresses and variabilities at each potential failure site. The stress at each failure site is obtained as a function of environmental and usage conditions, as well as of product geometry and material properties. Thus, the approach proactively incorporates reliability in the design process by establishing a scientific basis for evaluating new materials, structures, and technologies, using well-designed tests, screens, safety factors, and acceleration transforms.

Traditional reliability assessment techniques heavily penalize new materials, structures, and technologies because of insufficient failure data. This approach, based on fear of the unknown, rather than on any science-based analysis, discourages change, hindering reliability enhancement. The physics-of-failure approach, on the other hand, is based on generic failure models that are as effective for new materials and structures as they are for existing designs. The approach encourages innovative design through the use of realistic reliability assessment. This paper discusses the physics-of-failure approach to 
design, reliability modeling, testing, and screening of MCMs that has been implemented in CADMP Software at the CALCE Electronic Packaging Research Center at the University of Maryland.

Table 1: Results of 1987 SINCGARS NDI Candidate Test

\begin{tabular}{ccc}
\hline Vendor & $\begin{array}{c}\text { MIL-HDBK-217 } \\
\text { MTBF }\end{array}$ & $\begin{array}{c}\text { Actual Test } \\
\text { MTBF }\end{array}$ \\
\hline A & 811 & 98 \\
B & 1269 & 74 \\
C & 1845 & 2174 \\
D & 2000 & 624 \\
E & 2000 & 51 \\
F & 2304 & 6903 \\
G & 2450 & 472 \\
H & 2840 & 1160 \\
I & 3080 & 3612 \\
\hline
\end{tabular}

MTBF requirements 1250 hours with $80 \%$ statistical confidence.

\section{SOFTWARE TOOLS}

Computer-Aided Design of Microelectronic Packages (CADMP) is a set of integrated software programs that can be used to design and assess integrated circuit (IC), hybrid, and multichip module (MCM) packages. CADMP software consists of the various tools including: mission profile, geometry design, material selection, die placement, thermal analysis and calculation of thermal resistance, thermo-mechanical stress analysis, life prediction based on potential failure mechanisms and associated failure sites, reliability assessment and test development, screening, derating methods, material database, environment database, and component database. CADMP can improve assessment of component reliability during the design phase; evaluation of new materials, structures, and technologies; assessment of packages designed by other software programs or manufacturers; the development of sciencebased tests, screens, and derating methods; and costeffective product development. The potential users of this software include designers, manufacturers, and testers of IC, hybrid, and MCM packages; com, nies that use IC, hybrid, and MCM packages for manufacture; and agencies that assess, evaluate, test, or define specifications and requirements for IC, hybrid, and MCM packages or circuit cards and equipment that use these packages.

\section{Libraries}

Databases are provided for package element materials, environments, tests, screens, components, and failure mechanism models.

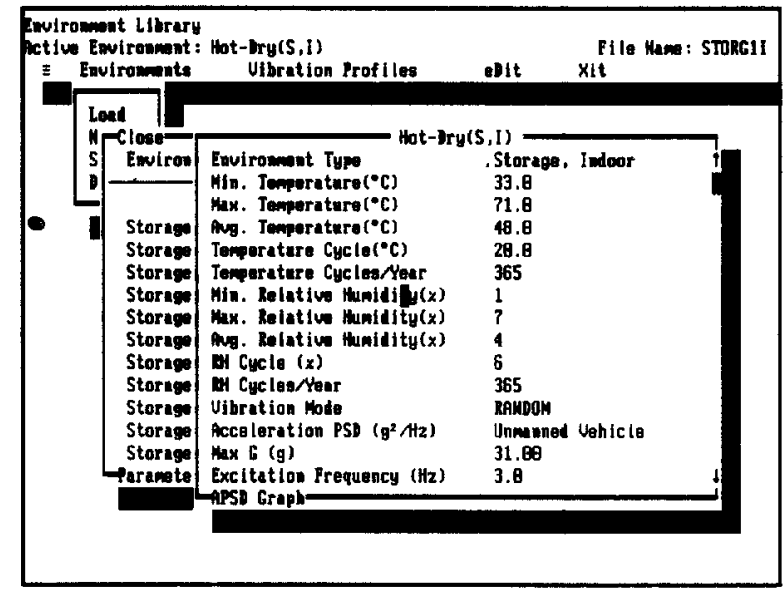

Figure 1 Parameters used by the environmental library

The package element materials in the database include die or chip, first-level interconnects (including wire bond interconnects, tape-automated bonds, flip-chip, flip-TAB and $\mathrm{HDI}$ ), device packaging materials include die attach, substrate, substrate attach, case, lid, lid seal, lead seal, and lead). The libraries can be accessed during the design process and serve as ready references to aid the design team. The user can edit the libraries to add additional environments, tests, screens, materials, components, or failure models.

The environment library includes complete descriptions of common electronic environments in terms of their characteristic load parameters - temperature (minimum, maximum, and average), magnitude and number of temperature cycles per year, relative humidity or $\mathrm{RH}$ (minimum, maximum, and average), RH cycle, vibration load (including the acceleration power spectral density, frequency, waveform, and vibration mode), maximum acceleration load, wind, rain, snow, sand/dust, salt spray, and radiation (Figure 1). If an environment required by the user is not available in the library, the user can create a new one by inputting the values of required load parameters.

The test and screen library includes a complete description of common tests and screens. Each is described in terms of its characteristic stresses, which include temperature, relative humidity, pressure, electrostatic discharge, radiation, and refined forces (e.g., bond pull or die shear). The user can select the stresses that will be part of his test or screen requirements (Figure 2). 
Each stress that is part of the test or screen is characterized by a set of parameters, which include maximum, minimum, dwell at maximum, dwell at minimum, ramp time maximum-to-minimum, ramp time minimum-to-maximum for temperature, relative humidity, and pressure; pulse time, current, and voltage for electrostatic discharge; mode, max acceleration, and excitation frequency for vibration; and energy, dose, dose rate, and pulse width for radiation. If a test or screen required by the user is not available in the library, the user can create a new one by inputting the values of required load parameters.

The material library stores properties for various package element materials. These properties provide a basis for

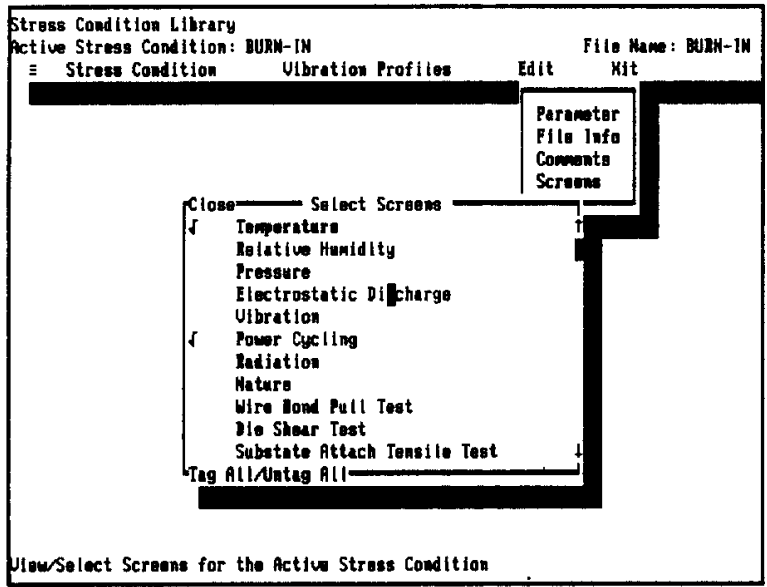

Figure 2 The user can select the stresses that will be a part of the test or screen.

selecting of materials for different package elements, and also form inputs for the physics-of-failure models for reliability assessment.

Material properties can vary significantly, depending on the ambient conditions at which they were measured. Therefore, the material library provides a framework to store temperature, moisture, frequency, and strain rate dependencies of material properties.

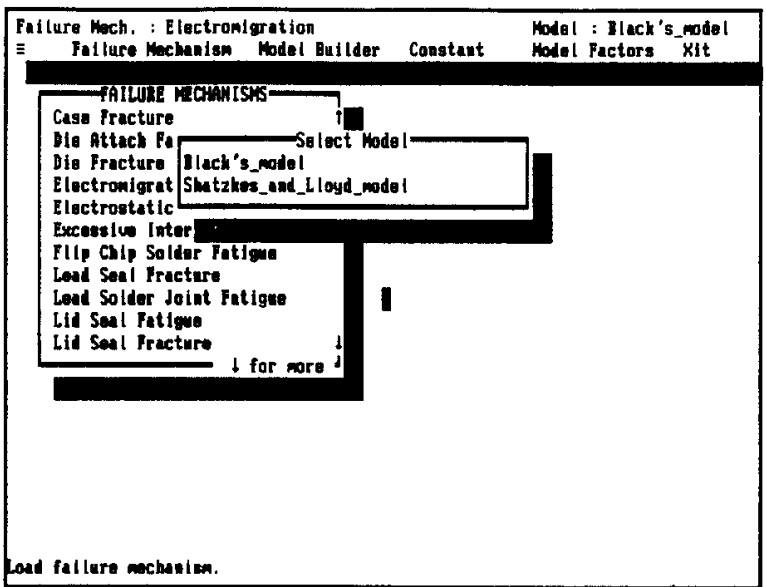

Figure 3 The failure model library contains algorithms for potential electronic failure assessment.

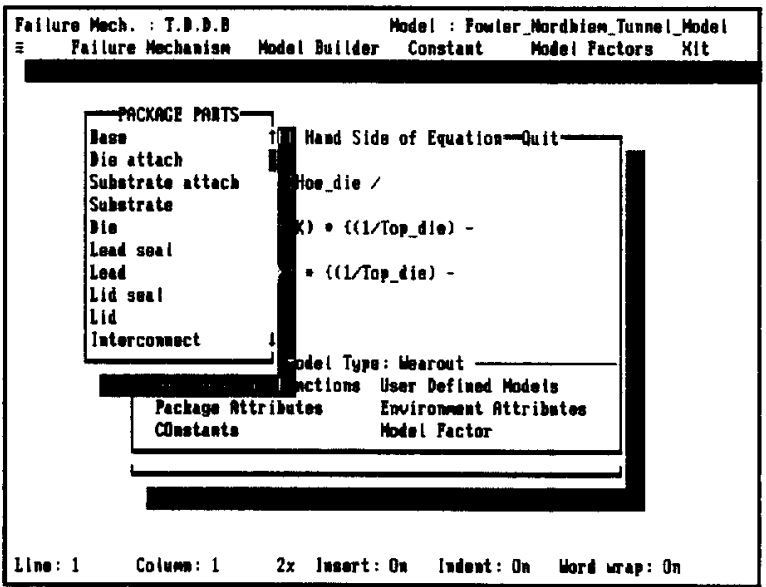

Figure 4 The user can also use alternative models for reliability evaluation by inputting them into the failure-model library.

The component library provides information on active and passive circuit elements within a package, including die or chip, resistors, and capacitors. Each component is characterized in terms of such parameters as physical dimensions and electrical specifications. Components selected from the library can be placed on the substrate to represent the packaging configuration quickly and to obtain required information for subsequent reliability assessment.

The failure-model library provides a systemized approach to creating, editing, and accessing information pertaining to potential electronic failure mechanisms (Figure 3). The failure-model library contains algorithms for potential electronic failure assessment; these are accessed by a parser that can check out and read a failure model from 
the library, assign numerical values to the parameters in the failure model, and collect all the reliability values for the complete electronic package. The library also includes constants, variables, model factors, assumptions, validation efforts, and references associated with the model. The user can also generate alternative models for reliability evaluation by inputting them into the failure model library (Figure 4). This ability to modify models also ensures that the failure-model library can be continually updated to reflect state-of-the-art advances in failure mechanism modeling.

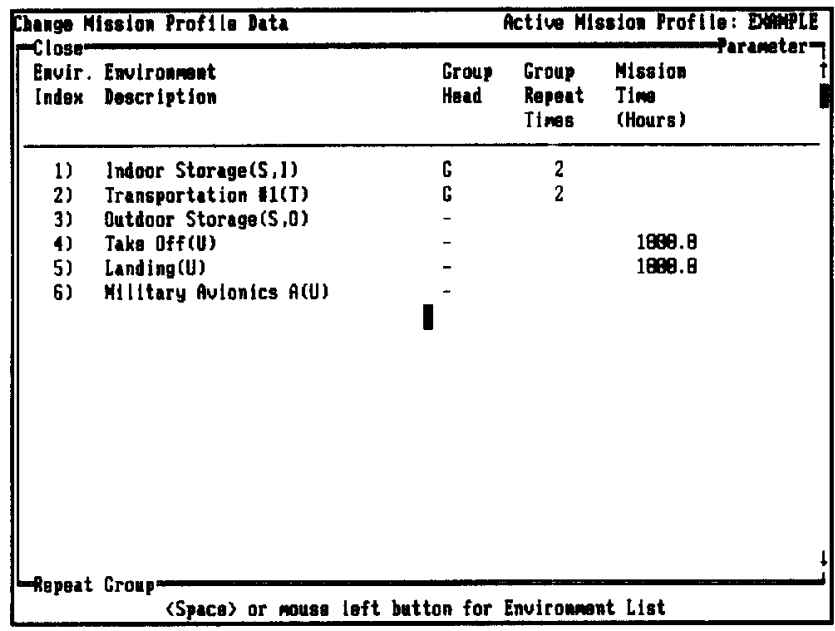

Figure 5 The mission profile tool allows the user to specify the environment, test, and screen durations to which the package will be exposed during the mission life.

\section{Mission Profile}

Mission profile refers to the magnitude and duration of all the loads the package is subjected to during its life-cycle, including environmental, testing, and screening loads. The mission profile tool allows the user to specify the environment, test, and screen durations to which the package will be exposed during mission life (Figure 5). Mission profile information is used by the failure-model library to evaluate the dominant failure mechanisms in the device architecture under the specified environmental, test, and screen loads.

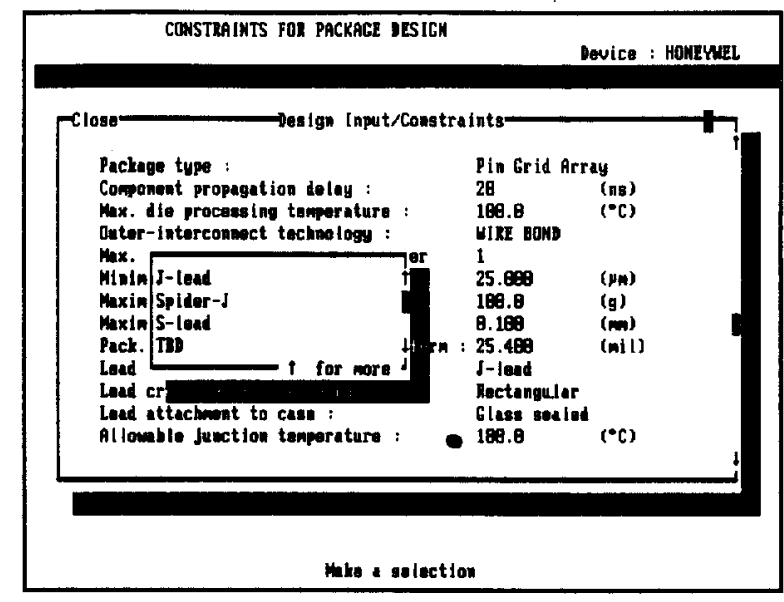

Figure 6 The constraints tool enables the design team to specify the broad parameters for MCM design.

\section{Constraints}

The constraints tool enables the design team to specify the essential features of MCM being designed. Examples of constraints include the number of package L/Os; allowable values of electrical parameters; and package architecture(Figure 6). The constraints can also include the non-availability of certain package architectures because of manufacturing limitations.

\section{$\underline{\text { Package Architecture }}$}

The package architecture tool provides the ability to specify the geometry and materials of a package. The user can start with a new design, or modify a previously designed package from the CADMP parts library. Design aids are provided to help the user in the selection of inner (die-to-package) interconnect technology, substrate, package-to-board mounting technology and package type e.g., dual in-line package (DIP) , quad flat pack (QFP), pin grid array (PGA), or multi-in-line package (MIP). The user can specify the electrical parameters and physical dimensions of the active and passive elements to be packaged in the multichip module. Finally, the package is completely described by specifying the dimensions and materials for each package element, including the element attach, substrate, substrate attach, lead, lead seal, lid, lid seal, case, and interconnects. (Figure 7 is a graphic display of an electronic package modeled in CADMP.) 


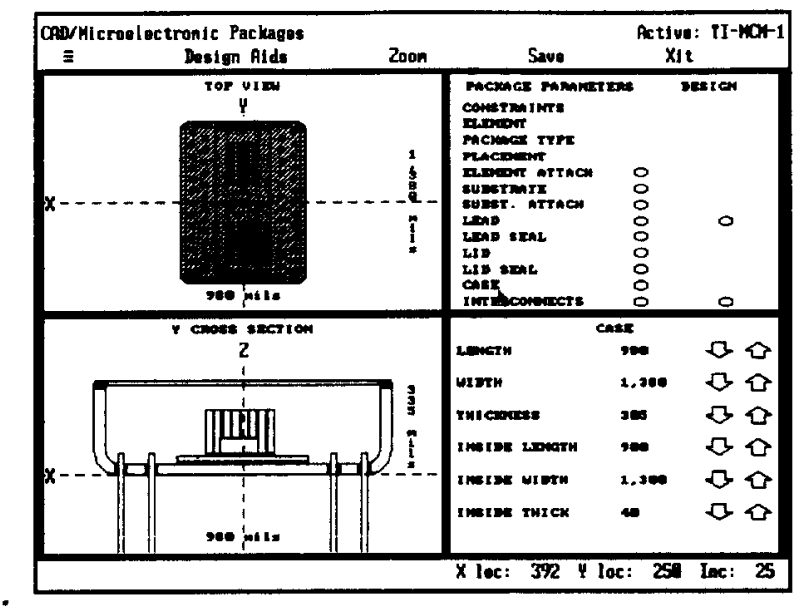

Figure 7 A graphic display of an electronic package modeled in CADMP.

\section{Thermal stress analysis}

For the given package configuration - that is, geometry, materials, and power dissipation of circuit elements CADMP performs a finite-difference-based thermal stress analysis of the package. The boundary conditions of thermal analysis are specified in terms of temperatures along the sides, bottom, and top of the package. The thermal analysis results are displayed as a map of the temperature distribution in various package elements. (Results of a thermal analysis are displayed in figure 8.)

\section{$\underline{\text { Reliability }}$}

The reliability analysis in CADMP calculates the time to failure for dominant failure mechanisms in the multichip module. The failure mechanisms are then

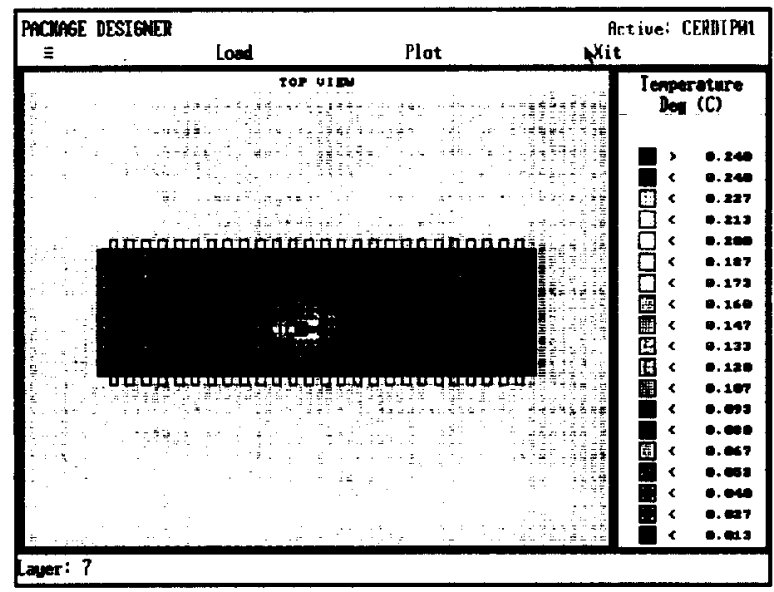

Figure 8 Results of a thermal stress analysis.

ranked in terms of their time to failure. The failure models used to evaluate each mechanism can be selected from the failure-mechanism library or can be user-specified, depending on the application. The reliability information can be used to assess whether the package will survive for the designed-for life. If the time to failure for the mechanism with the lowest time is less than the desired mission life, then the sensitivity of the failure mechanism to design parameters can be iteratively evaluated until system reliability goals are met.

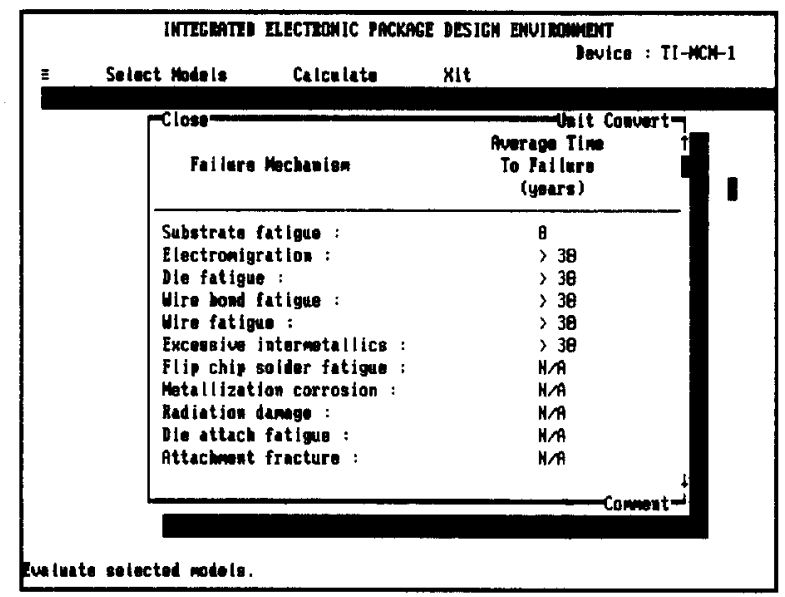

Figure 9: The failure mechanisms are ranked in terms of their time to failure.

\section{Screening, Qualification, and Derating}

Reliability analysis of the package in terms of potential failure mechanisms can provide a scientific basis for establishing screening, qualification, and derating guidelines. Screening is the process by which defective parts resulting from improper or out-of-control manufacture and assembly processes are detected and eliminated from a production batch. The principle involves only inducing latent defect failures in a population of parts that already has a percentage of "weak" parts, without reducing the reliability in the population of "strong" parts. Physics-of-failure based models can potentially be used to determine science-based screening plans, including determination of type, magnitude, and duration of the screening load required to detect or precipitate manufacturing flaws.

Qualification refers to the use of tests or screens, in which loads higher than actual operating loads may be used to reduce the testing time. Physics-of-failure models provide information on what loai can accelerate a particular failure mechanism and the affect of increasing the load on the time-to-failure above the normal operating load. The idea is to address use condition failure mechanisms during testing and screening. Thus, physics-of-failure tools can be used to determine the type, magnitude, and duration of accelerated test loads, and to extrapolate test results to life under normal operating conditions. 


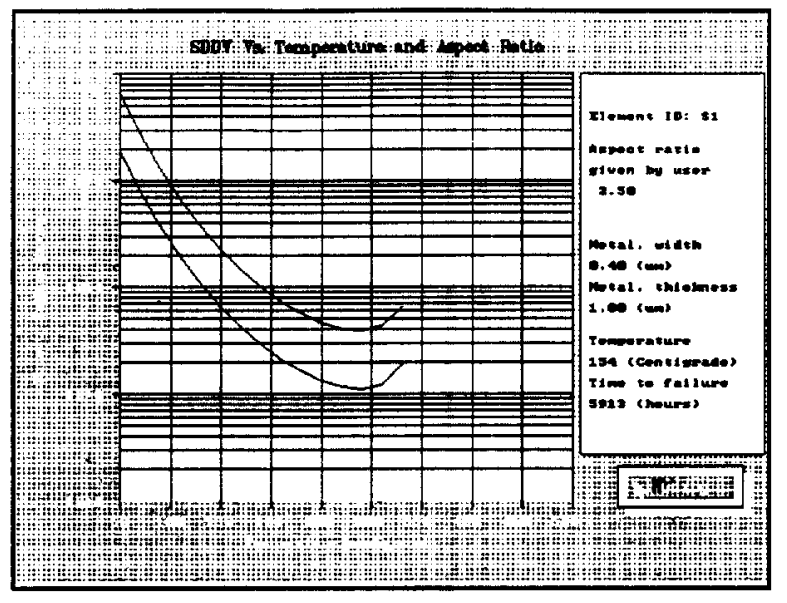

Figure 10: The derating tool derives derating curves specific to the multichip module architecture, for each failure mechanism.

Derating is based on the concept that operating electrical,thermo-mechanical, and chemical stresses accelerate failures predictably, and that controlling these stresses will improve reliability. The derating tool derives derating curves specific to the multichip module architecture for each failure mechanism in order to evaluate the effect of various stresses on the $\mathrm{MCM}$ operating life (Figure 10). The cumulative effects of various stresses acting on the multichip module are also evaluated to determine the levels to which the stresses must be reduced to achieve failure- free configurations for the desired mission life (Figure 11).

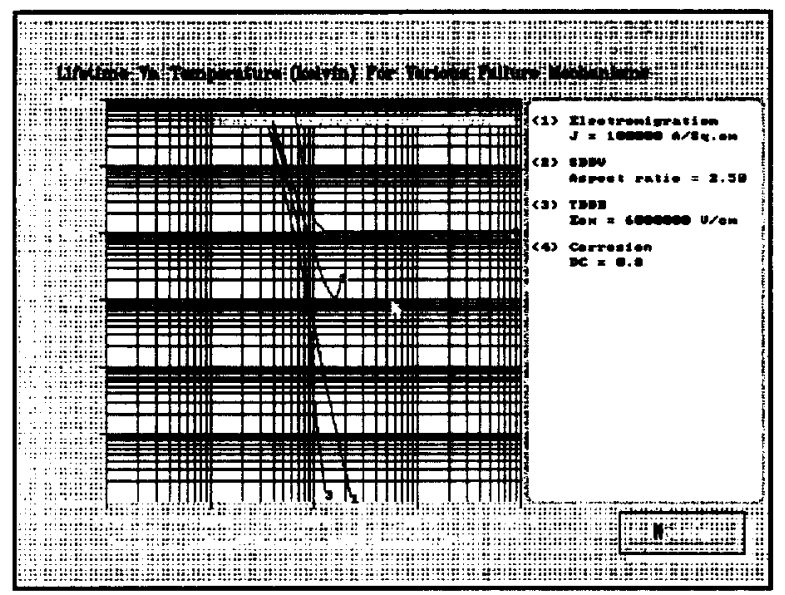

Figure 11 The cumulative effects of various stresses acting on the multichip module are evaluated.

\section{SUMMARY}

A physics-of-failure approach and the associated CADMF Software assist cost-effective MCM designs scientific consideration of reliability during the design phase; evaluation of new materials, structures, and technologies; assessment of packages designed by different manufacturers; development of science-based tests, screens, and derating methods. The tools can also be used to assess reliability, of single chip package designs, including plastic encapsulated microcircuits (PEMs). The development and validation process for CADMP Software continues. At present, the software is being exercised by over forty government and industry organizations, and feedback is being used to ready the software for commercialization. This project is funded by a grant from the US Army Research Laboratory.

\section{ACKNOWLEDGEMENTS}

The CADMP Software has been developed jointly by a team of researchers and computer programmers at the CALCE Electronic Packaging Research Center, University of Maryland. The programming group includes Michael Osterman, Xiaoyue Yang, Xuning Shan, Dave Beaudet, Xuping Xiang, and Yisheng $\mathrm{Wu}$, in addition to many who have contributed to the software in the past.

\section{REFERENCES}

1. M. Cushing, D. Mortin, T. Stadterman and A. Malhotra, Comparison of Electronics-Reliability Assessment Approaches, IEEE Transactions on Reliability, Vol. 42, No. 4, pp. 542-546, December 1993.

2. P. Lall, M. Pecht, An Integrated Physics-of-Failure Approach to Reliability Assessment, Advances in Electronic Packaging ASME EEP,Vol.4-1, 1993.

3. M. Pecht, D. Barker, A. Dasgupta, An Approach to the Development of Package Design Guidelines, Intern. J. of Microcircuits and Electronic Packaging, Vol. 16(3), pp. 217-240, 1993.

4. M. Pecht, V. Ramappan, Are Components till the Major Problem: A Review of Electronic System and Device Field Failure Returns, IEEE Trans. CHMT, 15(6), 1160-1164, 1992.

5. P. Lall, M. Pecht, and E. Hakim, "Computer-Aided Reliability Simulation, Design and Assessment of MCMs: A Physics of Failure Approach", 1993 Advanced 
Microelectronics Technology Qualification, Reliability, and Logistics Workshop, Denver, CO, August 24-26, 1993.

6. P. Lall, C. Rust, X. Shan, and E. Hakim, "ComputerAided Design and Reliability Assessment of Microelectronic Packages", ELECTROSOFT 93, Southampton, United Kingdom, July 6-8, 1993.

7. P. Lall, C. Rust, E. Hakim, "Physics of Failure Based Computer Tools for Design of Microelectronic Packages", 2nd ESA Electronic Components Conference, Noordwijk, The Netherlands, May 24-28, 1993. 